CAHIERS DE

NARRATOLOGIE
Cahiers de Narratologie

Analyse et théorie narratives

$7 \mid 1996$

Mélanges espace \& temps

\title{
Le chiasme, notamment chez Albert Camus
}

« Le Langage est la maison de l'Etre » M. HEIDEGGER

Michel Granet

\section{(2) OpenEdition}

Journals

Édition électronique

URL : https://journals.openedition.org/narratologie/11761

DOI : 10.4000/narratologie.11761

ISSN : $1765-307 X$

Éditeur

LIRCES

Édition imprimée

Date de publication : 1 janvier 1996

Pagination : 15-22

ISSN : 0993-8516

Référence électronique

Michel Granet, «Le chiasme, notamment chez Albert Camus », Cahiers de Narratologie [En ligne], 7 |

1996, mis en ligne le 23 février 2021, consulté le 05 mai 2021. URL : http://journals.openedition.org/ narratologie/11761; DOI : https://doi.org/10.4000/narratologie.11761

Ce document a été généré automatiquement le 5 mai 2021.

Article L.111-1 du Code de la propriété intellectuelle. 


\title{
Le chiasme, notamment chez Albert Camus
}

\author{
« Le Langage est la maison de l'Etre » M. HEIDEGGER
}

Michel Granet

R. Bernard Pingaud écrivait récemment: "en décembre 1959, interrogé par un journaliste américain qui lui demandait ce que la critique avait négligé dans son œuvre. Camus répondait : « la part obscure, ce qu'il y a d'aveugle et d'instinct en moi »... Là est sans doute la clé de son pouvoir, dans cette part obscure qui exige qu'on l'interprète et qui résiste à toutes les interprétations ». Résistera-t-elle, cette part inconsciente, à ce que la grande rhétorique poétique grecque nous a confié de plus précieux parmi les figures de style, le chiasme? Nous pouvons peut-être résoudre cette énigme, si d'abord en respectant le merveilleux de la culture grecque, nous purifions notre esprit critique littéraire par la révélation des "petits mystères » du cycle de mots à l'échelle de la phrase, du chapitre ou de l'œuvre ; si ensuite nous nous initions au « grand mystère » du chiasme anacycle de mots proprement dit, aux trois échelles susdites; si enfin nous nous perfectionnons par le «mystère du sceau» chiasmique imposant son empreinte époptique à l'échelle du récit, si bien que d'objet énigmatique éclairé devenu méthode, il nous conduise à une rhétorique restreinte où resplendira, à la limite, l'anacycle de lettres.

2 «Purifions» d'abord notre esprit critique littéraire par un exemple de cycle homophonique tautologique ${ }^{1}$, où deux mots répétés dans un même ordre, conservent chacun la même fonction à l'échelle de la phrase, dans la première Partie, au chapitre I : «... à l'asile, elle (maman) pleurait souvent... à cause de l'habitude... elle aurait pleuré si on l'avait retiré de l'asile. Toujours à cause de l'habitude ». Cette phrase est structurée comme un quatrain horizontal : pleurait, habitude, pleuré, habitude, qui équivaut à quatre rimes riches verticales alternées et tisse ainsi la poésie dans la prose. Du point de vue de la situation de l'état, le «tout le monde » étranger domine l'« autre monde » étrange du pouvoir entier d'être soi libre; comme du point de vue du discours littéral du savoir qui régit le rapport Sujet-Objet, l'objet (pleur/habitude/qui appartient aux/sentiments distingués/: loger, nourrir sa mère, causer avec, savoir son âge. la voir morte, se 
recueillir sur son cercueil et pleurer, et tant d'autres que Camus flétrira dans "L'étranger » : «j'ai eu une envie stupide de pleurer: parce que j'ai senti combien j'étais détesté par tous ces gens-là, et dans "Le mythe de Sisyphe" sous le terme de "sensibilité absurde ») domine le sujet Meursault: comme du point de vue du paraboler littéraire de la vérité, le par (abo) ler littéral le domine; comme du point de vue ontologique l'être là-devant domine l'être-au-monde.

3 A l'échelle de l'œuvre; 1 ère Partie, chapitre I: tic - 1-V-VI : automate, tic - $2^{\text {ème }}$ Partie, chapitre I; tic- $2-\mathrm{V}$ : automatique, quatre rimes hypophoniques alternées confirment cette équivalence, cette ambivalence, cette orthodoxie, comme à l'échelle de la $2^{\text {ème }}$ partie, chapitre V, quatre rimes anaphoriques suivies respectivement «mécanique " et "machine ", expriment la dernière torture physique et morale et le dernier sacrifice humain de «Meursault» sous le "soleil » ( $1^{\text {ère }}$ partie, chapitres I et VI) et sous la " guillotine " ( $2^{\text {ème }}$ partie, chapitre V) et réactivent la métaphore surréaliste : «Soleil, cou coupé ». «Il n'y a pas d'issue » : tel est le discours conscient du savoir où le réel se répète à la même place, fermé temporellement, sans autre possibilité d'ouverture.

Maintenant, initions-nous, en premier lieu, par un exemple d'anacycle homophonigue chiasmique où deux mots répétés dans deux ordres inverses, fonctionnent dans deux sens différents, à l'échelle du paragraphe, $2^{\text {ème }}$ partie, chapitre I : « mais à travers la table, il (le juge) avançait déjà le Christ sous mes yeux et s'écriait... :... « comment peux-tu ne pas croire qu'il a souffert pour toi?... Et au bout des onze mois qu'a duré cette instruction...: " c'est fini pour aujourd'hui Monsieur l'Antéchrist ». Ce paragraphe est structuré comme un quatrain horizontal: christ, tu; anti-tu (Monsieur) antéchrist, quatrain horizontal qui équivaut à quatre rimes verticales embrassées qui, comme telles, érotisent ainsi la poésie dans la prose, symétriques par rapport à une droite ${ }^{2}$, comme deux dragons-serpents qui se mordent la queue et expriment l'équivoque, l'ambiguïté et le paradoxe. Symétriques par rapport à un point, comme un cercle tordu en huit, elles constituent le chiasme, de la lettre Ki grecque ; apparue avec Hermogéne de Tarse au II ${ }^{\text {ème }}$ siècle après J. C, à l'époque de la seconde sophistique, quand l'atticisme avait peur de l'asianisme : le taï ki chinois, le dorje hindou rappellent le même symbole que le caducée athéno-romain et que le triskel gaulois, syncrétisés par le chrisme chrétien, synthétisant une perspective de détour fermé à une perspective de retour ouverte dans une anamorphose littéraire, ce qui empêche dans tous les cas de réduire le chiasme à une simple antithèse $e^{3}$. Du point de vue de la situation de l'état, un "tout le monde " étranger domine un autre «tout le monde » étranger ; comme du point de vue de la par (ab) ole vide qui régit le rapport Objet/Maître et Objet maîtrisé, l'objet maître, (le délire de l'avocat général qui se mégalomanise en procureur et intègre celui du juge, mauvais fonctionnaire, machine du Christ ; celui des journalistes qui amalgament le soi-disant matricide de Meursault avec le parricide ; celui de l'aumônier, professionnel de la foi, qui a peu de foi dans sa profession) domine l'objet maîtrisé Meursault; ce dernier et le juge s'objectivent en miroir ${ }^{4}$ comme le par (abo) ler vide de l'un (cœur de criminel 2 III, âme criminelle 2 IV) domine le par (abo) ler vide de l'autre (2 IV penché sur mon âme, mon cœur fermé) : le message est donc identique mais le mythe est inversé: Antéchrist et Christ. C'est le mécanisme hégélien de la conscience malheureuse, de la belle âme qui croit agir selon la loi $d u$ cœur mais reste inconsciente du désordre sociosexuel qu'elle propage dans la cité, en refoulant sous le culte de la mère son propre désir œdipien d'inceste maternel, en violant sadiquement et moralement Marie sous les yeux de Meursault ; comme du point de vue ontologique un être parmi les autres et son on-dit 
domine un autre être parmi les autres et son on-dit (c'est-à-dire son «bon » sens, son «bon » goût, sa « bonne » volonté).

5 A l'échelle de la phrase, l'avocat général qui se voit déjà procureur n'affirme-t-il pas : «j'en (crime prémédité) ferai la preuve... et doublement sous l'aveuglante/clarté des faits d'abord et ensuite dans l'éclairage/sombre que me fournira la psychologie ", en fait l'imaginaire narcissique du corps propre d'un procureur paranoïaque qui s'accepte très mal dans le corps morcelé d'un avocat général. Tel est le par $(\mathrm{ab})$ oler vide avec qui : « tout est vrai et rien n'est vrai » (2 III)... Si... il m'avait vu pleurer... non... « s'il avait vu que je ne pleurais pas »... « Non ». «Le public a ri »... «Enfin, est-il (Meursault) accusé d'avoir enterré sa mère ou d'avoir tué un homme?»

2. «Initions »-nous, en second lieu, par un autre exemple d'anacycle homophonique chiasmique de la phrase dans la première partie chapitre $6:$ : Masson a dit qu'on avait mangé très tôt, et que c'était naturel, parce que l'heure du déjeuner c'était l'heure où l'on avait faim. Je ne sais pas pourquoi cela a fait rire Marie ». En effet Marie n'a pas l'habitude d'avoir "envie» à l'heure, comme nous le verrons tout à l'heure. Maintenant, la seconde partie du chiasme in présentia qui exprime un "retour $»^{5} \mathrm{du}$ sujet dominant l'objet, doit être précédé de la part du lecteur actif par une première partie in abstentia qui exprime un détour ${ }^{4}$ de l'objet dominant le sujet "on a faim à l'heure » refoulé dans l'inconscient et qui nous ramène au discours du savoir, au par (abo) ler, quatrain horizontal équivalent à quatre rimes verticales embrassées. Mais à l'échelle de la première partie se manifeste un cycle homophonique tautologique : « ... samedi,... j'ai retrouvé dans l'eau Marie Cardona... dont j'avais eu envie à l'époque... Le soir elle est venue chez moi... (1 II)... samedi... j'ai eu très envie d'elle... c'était bon de sentir la nuit d'été couler sur nos corps bruns... (1 IV)... ce matin, dimanche, j'ai eu encore envie d'elle... (1 IV)... dimanche j'en avais envie... j'ai senti ses jambes autour des miennes et je l'ai désirée... (1 IV)». Dans ce quatrain anaphorique équivalent à quatre rimes verticales suivies, l'alternance samedi/dimanche, érotise plus que jamais la prose, non par sa forme d'expression, cette fois, mais par sa forme de contenu. Du point de vue de la situation de l'état, l'« autre monde » étrange du pouvoir entier d'être soi libre domine le « tout le monde » étranger; comme du point de vue de ce discours littéral intermédiaire qui régit le rapport Sujet/objet, le Sujet/envie/de/Meursault, qui peut osciller entre vérité et tromperie, domine l'objet/samedi/soir/dimanche/matin qui peut osciller entre exactitude et erreur; comme du point de vue du paraboler littéraire qui régit le rapport avec le par (abo) ler littéral et le domine; comme du point de vue ontologique l'éveil de l'être au monde commence à dominer l'être là devant de la ronde des heures et de jours. Ce discours littéral est bien intermédiaire entre le par (abo) ler du discours $d u$ savoir, le par (abo) ler de la parole vide et le paraboler de la parole pleine, trompeuse ou vraie, toujours au moins double, dont la voix va bientôt se faire entendre.

3 - Initions-nous en troisième lieu, à l'exemple suivant d'anacycle homophonique chiasmique à l'échelle de la phrase in praesentia/in abs entia: "je la tapais, mais tendrement ». La Mauresque aurait préféré que Raymond dise : «je m'attendrissais en la tapant » ce qui l'aurait peut-être conduit à ne plus la battre « jusqu'au sang ». De même quand Raymond dit in praesentia qu' " il avait encore un sentiment pour son coït», la Mauresque aurait préféré que Raymond fasse «le coït par sentiment». Qu'est-ce qui conduit le lecteur conscient à cette lecture perspicace de l'inconscient ? C'est l'oxymoron ironique qui constitue le premier membre in praesentia de chaque chiasme. Le comble, c'est que Raymond répète en cycle anaphorique quatre fois "tromperie ", tout en 
cachant la parabole pleine vraie de bonne foi derrière la parole pleine trompeuse de mauvaise foi en la refoulant ou la forclosant dans son inconscient sexuel. Le patron emploie la voix inverse: il refoule ou forclot derrière sa parabole pleine vraie sa parabole pleine trompeuse: il propose à Meursault un «changement de vie» in praesentia, alors qu'in abstentia il s'agit d'« une vie de changement », puisqu'il veut transformer Meursault en voyageur de commerce parisien!

Mais à l'échelle du paragraphe de la $1^{\text {ère }}$ Partie du chapitre VI l'anacycle homophonique chiasmique le plus important se manifeste in praesentia .... « Raymond a porté la main à sa poche-revolver... Alors je vais l'insulter et quand il répondra je le descendrai »... «Non... Si l'autre... tire son couteau, je le descendrai »... Raymond m'a donné son revolver ». Ce quatrain horizontal équivaut encore à quatre rimes riches poétiques verticales embrassées qui érotisent la prose. Du point de vue de la situation de l'état d'âme, l'« autre monde » étrange du pouvoir entier d'être soi libre domine un « autre monde " étrange du pouvoir entier d'être soi libre; comme du point de vue de la parabole pleine qui régit le rapport Sujet maître et sujet maîtrisé, le Sujet maître, (le « je le descendrai» de Raymond) domine l'autre sujet (le «je le desccendrai »... si de Meursault) : le message est inversé mais l'histoire est identique : c'est celle d'un revolver. L'inversion du message ressemble à l'histoire du juif polonais qui dit à son compagnon : "Pourquoi me dis-tu que tu vas à Cracovie, pour me faire croire que tu vas à Varsovie alors que tu vas vraiment à Cracovie? » Faire le vrai pour faire faire le faux telle est la conduite de Raymond à qui Meursault pourrait dire: "Pourquoi me donnes-tu ton revolver pour me faire croire que tu ne le descendras pas, alors que tu veux que je descende l'Arabe? ? La structure de la parabole pleine même trompeuse est l'inverse de celle de la par (ab) ole vide; comme du point de vue ontologique un être avec les autres domine encore un autre être avec les autres.

9 A l'échelle de la phrase se manifeste l'anacycle homophonique chiasmique in praesentia/ abs entia de la $2^{\text {ème }}$ partie au chapitre $1:$ pendant sa détention, Meursault interrogé par le juge, entend le greffier qui se trompait de touches, quand il affirme qu'il aimait sa mère comme " tout le monde ». Ici l'acte raté du greffier refoule une parabole silencieuse réussie: il croit «toucher à la tromperie» de Meursault. Ce même acte raté réactive la perspicacité du lecteur. A la même échelle phrastique, Meursault dit dans la $2^{2 \mathrm{èe}}$ partie au chapitre IV : «j'ai trouvé que sa façon (à l'avocat général) de voir les événements ne manquait pas de clarté ». Le lecteur sursaute : c'est que Meursault, être avec les autres, n'a pas encore atteint le niveau d'être au monde et son pouvoir entier d'être soi libre. Il refoule encore parabole silencieuse qui demeure in abs entia : «j'ai trouvé que sa façon de voir les événements prouvait la clarté du manque de circonstances atténuantes » (le rôle du soleil, du vin... et de la provocation de Raymond, de l'amalgame criminel des journalistes.)

104 - «Initions-nous" enfin en quatrième lieu au dernier exemple d'anacycle homophonique chiasmique à l'échelle de l'œuvre : «A travers les lignes de cyprès... je comprenais Maman. Le soir devait être comme une trève mélancolique (1 I)... Le jour déjà plein de soleil m'a frappé comme une gifle (1 VI - 1)... Je ne sentais plus que les cymbales du soleil sur mon front (1 VI - 2)... J'ai pensé à Maman... Je comprenais pourquoi... Le soir était comme une trève mélancolique... Si prés de la mort, Maman devait s'y sentir prête à tout revivre... »L'œuvre entière est donc structurée comme un énorme quatrain horizontal : cyprès, soir, soleil, soleil-soir, si près équivalent à quatre rimes verticales embrassées. Disons plus : si nous remarquons que la locution «écrire une 
lettre » (1 III fin) résonne avec la locution «lettre écrite» (2 III fin) et se réfléchit dans une double symétrie par rapport à une droite et par rapport à un point (épanalepse); que la locution «envoyé la lettre (1 IV début)/résonne et se répète en parallèle avec la locution « reçu la lettre » (2 II début), nous constatons qu'un autre chiasme horizontal se recoupe avec le premier chiasme vertical pour structurer l'œuvre selon une resplendissante croix de Malte, si nous répartissons les XI chapitres en XII en divisant le 1 VI par deux = 1 VI 1 et 1 VI 2. Le chiasme vertical structure les forces du contenu, la lettre de l'être et l'horizontal les formes de l'expression, l'être de la lettre. Du point de vue de la situation de l'état d'âme, un "autre monde » étrange se complète dans un "autre monde " étrange ; comme du point de vue de la parabole pleine et vraie qui régit le rapport Sujet-Sujet, le sujet « Meursault » reconnaît définitivement le sujet « maman » $(2 \mathrm{~V})$ qui l'avait déjà reconnu du regard (1 I) : selon le message implicite inverse : «Je suis ta mère, $\mathrm{Tu}$ es mon fils - Tu es ma mère. Je suis ton fils. L'histoire du soir. Trève mélancolique reste identique. La parabole pleine vraie est structurée à l'inverse de la par (ab) ole vide. Du point de vue ontologique un être au monde avec les autres se reconnaît dans un autre être au monde avec les autres, de telle manière que, comme lui, il est si près de mourir à son échec qu'il est aussi prêt à revivre une nouvelle réussite et être enfin, vers les sommets, un Sisyphe heureux, qui reste là sans être las. Tel est le paraboler plein et vrai, équivoque, ambigu, paradoxal qui ouvre éternellement la réalité sur la possibilité, par un «retour» à partir d'un «détour", par un changement de perspective anamorphique.

111 - Maintenant «perfectionnons» en premier lieu notre esprit critique littéraire. La grande rhétorique grecque nous fournit chiasmiquement non plus seulement les figures et les tropes hypophrastiques du langage objet du récit, mais les concepts théoriques qui les recouvrent et permettent de définir une rhétorique restreinte de base, métaphore/ diaphore. La métaphore catachrésique de sens est une diaphore hétérophonique de sons (un signifié, deux signifiants) :

\section{(lumière) brûlante

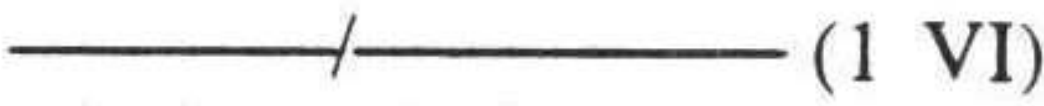 épée (pénétrante)}

refoule «lumière » et "pénétrante » et marque ainsi le temps de la fiction sexuelle «Meursault - Marie Cardona », tandis que 


\section{(début) malheur} DU

\section{porte (tribunal)}

refoule

« début » et «tribunal » et marque le temps de la narration textuelle qui divise le récit en deux parties. Ces deux métaphores catachrésiques, symptômes, en exprimant une " porte » et une « épée » surnaturalistes posent la question de la lettre de l'être et de l'être de la lettre.

Il en sera de même pour la figure contraire la diaphore hétérosémantique de sens qui est une métaphore homophonique de sons (deux signifiés pour un Signifiant) : "tirer ", homographique à "tuer", compte le temps qui passe de la fiction sexuelle, tandis que "air» de l'atmosphère et "air» des personnages compte le temps qui passe de la narration textuelle et divise le récit en deux parties.

La figure différente, la diaphore hétérosémantique de sens est une métaphore hypophonique de sons (deux signifiés pour un Signifiant): "coup »; hypophonique à "coupable", compte le temps qui passe de la fiction sexuelle, "tic", hypophonique à « automatique »; compte le temps qui passe de la narration textuelle et divise le récit en deux parties. Ces deux diaphores hypophoniques, pointes du désir, exprimant un « coup » et un « tic » surréalistes expriment ainsi le manque à être.

Il en sera de même pour la figure contraire la métaphore métonymique de sens qui est une diaphore hétérophonique de sons (un signifié pour deux Signifiants) l'étoile (à cinq branches) ${ }^{6}$ qui symbolise la deuxième partie marque le complexe de culpabilité et de castration par rapport à l'étoile (à six branches) ${ }^{7}$ de la jouissance qui symbolise la première partie. Elles comptent le temps de la narration textuelle en tant que partie du tout du décor; les «deux chevalets en forme de * «qui supportent le cercueil de la maman et supporteront peut-être plus tard celui de Meursault, ne peuvent qu'avoir la structure suivante: ${ }^{*}(6)+*(6)=$ XII chapitres implicites, si le sixième chapitre de la première partie est divisé en deux. Ils comptent le temps de la fiction sexuelle. Mais si ${ }^{*}=$ $\mathrm{X}+\mathrm{I}=\mathrm{XI}$ chapitres explicites, il compte le temps de la fiction textuelle. Si les structures chiasmiques hypérophrastiques peuvent être inconscientes, les hypophrastiques ne peuvent être que conscientes: quand l'auteur n'est pas jouissant sexuellement, c'est qu'il est jouissant textuellement: la jouissance phallique est remplacée par la jouissance calamique.

15 Le chiasme rhétorique théorique qui rend compte des figures et des tropes à l'échelle hypophrastique appelle son semblable à l'échelle hypérophrastique de toute l'œuvre : le prologue (1 I) et l'épilogue ( $2 \mathrm{~V}$ ) qui en rhétorique traditionnelle expriment le pathos de l'auditeur, expriment ici l'ithos du lecteur, tandis que la narration (1 II - VI-1) et la confirmation (1 VI-2 - IV), qui en rhétorique traditionnelle expriment l'ithos de l'auditeur expriment ici le pathos du lecteur. Le chiasme théorique, c'est bien le chiasme rhétorique sans en avoir l'(ai)r, son sceau.

2 - «Perfectionnons » enfin en dernier lieu notre esprit critique littéraire en poussant à la limite le chiasme de deux mots en chiasme de neuf lettres: L'ETRANGER devient 
anacyclement REGNARTEL. N'oublions pas que Marie a pour patronyme Cardona : en espagnol « Renard». Or « renard » vient du germanique « regin » qui signifie conseil et de «hard» signifiant «fort». En vieux français «Regnart». «El» est un suffixe adjectif: comme dans «sensuel». "Regnartel» pourrait vouloir dire: conseiller judicieux c'est-à-dire Meursault à la fin du chapitre V de la $2^{\text {ème }}$ partie. Si bien que le titre suggère une parabole pleine et vraie où l'histoire est identique mais le message est inversé. L'auteur dit au lecteur: tu as place dans ma parole. Je suis ton récit, l'ETRANGER. Le lecteur répond à l'auteur : ma parole est à ta place. Tu es mon récit REGNARTEL. Le titre de IX lettres résume chiasmiquement XI chapitres.

Ainsi la parabole pleine vraie, la parabole pleine trompeuse, le discours intermédiaire, la parole vide, et le discours du savoir ont confirmé tour à tour le chiasme comme structure élémentaire de la signification dans "L'étranger» d'Albert CAMUS. Donc la part obscure, aveugle et "instinctive " du récit n'a pas résisté, quant à l'essentiel, à sa lecture perspicace. Mais les modernes que nous sommes sauront-ils reconnaître, selon la sentence maximale du philosophe Dominique JANICAUD ${ }^{8}$, que de toute façon le grand passé grec de la rhétorique nous attend au fond de l'avenir de tout chef d'œuvre littéraire moderne?

\section{NOTES}

1. Symbolisé depuis la plus haute antiquité par un dragon-serpent qui se mord la queue.

2. La droite-miroir apparaît dans le récit sous la forme d'une "glace » (1 II), d'un fond de « gamelle de fer» (2 II fin). Le texte rhétorique manifeste en lui-même l'allégorie qui institue son fonctionnement théorique.

3. L'antithèse est une déconstruction de sens qui peut conduire à l'aporie, à l'impasse. Le chiasme est une reconstruction qui conduit au passage du sens.

4. La droite-miroir apparaît dans le récit sous la forme d'une "glace» (1 II), d'un fond de " gamelle de fer » (2 II fin). Le texte rhétorique manifeste en lui-même l'allégorie qui institue son fonctionnement théorique.

5. Le récit pullule de : « détourner », se détourner »; « retourner », « se retourner ». De nouveau la métaphore métonymique empruntée au décor symbolise le fonctionnement du texte.

6. Cette superposition des deux étoiles -symboles en 6 et en 5- non seulement rappelle celle du carré magique 6 du soleil et du carré magique 5 de la terre dans le tao chinois, mais marque ici celle de l'étoile juive à six branches, symbole de la première partie du récit, avec Thomas Perez $\mathrm{au}$ "f feutre mou à calotte ronde et aux ailes larges ", et de l'étoile arabe à cinq branches, symbole de la seconde partie. Le thème de l'étoile fonctionne comme surréaliste.

7. Cette superposition des deux étoiles -symboles en 6 et en 5- non seulement rappelle celle du carré magique 6 du soleil et du carré magique 5 de la terre dans le tao chinois, mais marque ici celle de l'étoile juive à six branches, symbole de la première partie du récit, avec Thomas Perez $\mathrm{au}$ « feutre mou à calotte ronde et aux ailes larges », et de l'étoile arabe à cinq branches, symbole de la seconde partie. Le thème de l'étoile fonctionne comme surréaliste.

8. Lire «La métaphysique à la limite ", Dominique Janicaud et Jean-François Mattéi Collection Epiméthée, PUF - 1983, p. 13. 


\section{AUTEUR}

MICHEL GRANET

Université Paris X 